\title{
New Direction of Virtual Reality Game Development Combining Five-Senses Design
}

\author{
Mingen Yang ${ }^{1, a}$ \\ ${ }^{1}$ Guangzhou academy of fine arts City, Guangzhou 518000, China. \\ a547158723@qq.com
}

Keywords: Gaming, virtual reality, five senses.

\begin{abstract}
Along with the development of game industry, more and more game agencies and players start caring about new gaming styles. One of the latest approaches is to combine the virtual reality (VR) with the gaming experience. This paper will briefly introduce the evolution of gaming output devices, as well as the development of current VR games. Then, a new trend of VR gaming, Five-sense VR game, will be discussed, with two related examples. Five-sense VR games utilize five human senses to improve the gaming experience. According to the study, this kind of new game can effectively enhance the gaming experience and has a bright future.
\end{abstract}

\section{Introduction}

People are closely related to the game, Human beings could be defined as ' Homo Ludens, which means playing man and as Sohiler said, play is one of the most fundamental behaviors of human beings and it is the action which can make you feel the totality of life in the most evident manner [1]. This is because game is a form of basic human behavior and game is also a way to feel the life directly. people use physical, emotional, cognition ,creativity and sociality to play games. It can promote communication between human beings. Cause when people play the multi play game it can promote the relationship with friends and relatives. In addition to this. Every game requires an input device to operate and control it. The most common devices are keyboard, mouse, camera, scanner, pen, hand and voice input device. After that more and more new input devices came along, bringing the innovation of The Times. In recent years, Virtual Reality become a new type of input method. Comparing with some traditional way, the game experience which VR brings is completely different. it is not only a visual perception difference but also the interaction experience. In FPS games, VR is easier to be controlled than traditional devices like key board, mouse and wand. The result of a test is about female users who had never play the First Person Shooter games before is good. The accuracy of women who use VR are better than those who use common devices and VR gives them a sense of authenticity and a natural surrounding. [2] VR offers a variety of excellent experiences, which used in different areas including VR games, this is a very fresh area. And the development space of VR games is really large, and these excellent input experiences can be continuously optimized and improved. By this point, I think I can incorporate the five senses into it. Human beings attach the world with five senses. Through the sense of taste to taste the food, by the sense of smell to feel the fragrance of a flower, with the vision to see the shape of the item, through touch to feel how hard the rock is, with a hearing to listen to the sounds of nature. Because of the five senses, the image of the world becoming full. Likewise, the emergence of VR is because we want to make player experience more real, and we want to make a virtual image of the game plump, in addition to the immersive of VR and the feeling on the vision, we can also join other senses, such as the sense of touch, smell and hearing so I'm going to discuss this argument here. This paper is structured as following: First part reviews the related works on immersive VR games. Second part discusses the senses which can be put in to the games and why. Third part discusses the potential to develop VR games. The last part concludes this paper. 


\section{Two examples}

Immersive VR games are the fields which developing. There are excellent and rich varieties of VR games. However in this paper will be briefly introduce some games that which are revolutionary. The first one is created by Jefry Tedjokusumo, Steven ZhiYing Zhou and Stefan Winkler. They had found a Immersive Multiplayer Games With Tangible and Physical Interaction. It is a FPS game and it can be played by two players who use HMDs and a high-precision ultrasonic tracking system. [1] That is why Jumping is also tracked in this game so that the user has more options to avoid bullets. This game adds the interaction between game and people, which is one of its innovation points. Another game called 'Thinking Penguin' . Players used the Multi-modal Brain-Computer Interface to control it. It is described as the mental rehearsal of a motor act, without any overt motor output. It recorded electro-physiological brain signals and analysis them turn in to the way to control the direction of penguins in the game. The limitations of it is that it needs learning cost to learn how to use this control method. It is one of the reasons why it can't be a widespread way.

\section{Five senses with VR games}

Compared with the traditional operations, the advanced operations have the following advantages: 1. The accuracy of game operating was improved; 2 . The game experience become better; 3 . it is easy to learn how to operate. The most prominent of these is the gaming experience. The research staffs try different ways to increase the game experience. One of the attempts was to incorporate the five senses into game.

The human senses are divided into five different parts: vision, hearing, smell, taste and touch. One of the biggest difficulties is the sense of taste is so hard to be put in to games, because of the sense of taste has high cost. 1. The utilization rate is not high; 2 . Hard to spread. However the rest of these several senses are very easy to implement. why would these senses added in to the games? The answer is very clear. To increase the sense of reality.

People use five senses to perceive the world. For examples when people came to a lovely place, the first thing is about the vision. They could see the beautiful mountains, birds and trees. People could also hear birds chirp. At the same time, the sound of the flowing streams and the smell of the soil and flowers were exists in this space, then they would go to touch their nearest things, such as a tree, feeling the rough surface of the trees and they may took a sip of water from the stream, the impression about this place was completed. when people leaved this place to recall again, a complete image will be built in their mind. they can recall these feelings and memories. The image in our mind is resemble to the image we see in the game. It also the image developers want to present in players's sight. The game is virtual as people's memory. How developers make a person feel the surrounding of the game real? The only way is to attach the five senses of human.

\section{Huge potential to develop:}

Some of the powerful VR game engines are open source to the public so this show that the VR games have a broader space for development. [3]

Based on the control methods people commonly used. We can know that voice commands are related to people's auditory sense. The gestures and posture are related to people's visual sense.

The direct manipulation will often apply to the tactile sensation [1] and olfactory sensation [4]. All of these control methods refer to five senses of human and it is a low interaction costs. Especially subjects can achieve a nice grades [1] It is not just a sense of immersion to upgrade the game experience. The level of operation in the game can also be improved [2] . 


\section{Summary}

This paper mainly talks about the impact of the five senses on VR games, Comparing with traditional operations and the experience now VR games can bring to people, there is another direction to improve the experience of VR games ---- A game of five senses. The technology of five senses was to use different devices and install them into the players' equipment. Like their head trackers and wand trackers or their suits. The devices are conciseness just to improve on the original foundations. However, the technology of five senses was not flawless. Some of senses are hard to put into devices. and the other sense was worth to be develop. game developers need to keep researching the relationship between five senses and VR games.

\section{References}

[1] Joohun Lee, Haehyun Jung, Hyunggi Kim: Interactive Media Artworks as Play Therapy through Five Senses.(2013)

[2] Jefry Tedjokusumo, Steven Zhiying Zhou, Stefan Winkler: Immersive Multiplayer Games With Tangible and Physical Interaction.(2009)

[3] Rudolph P Darken, Perry Mcdowell, Erik Johnson: Projects in VR: The Delta3D open source game engine.(2005)

[4] Arito Mochizuki, Takashi Amada, Sayuri Sawa, Tadayuki Takeda, Shogo Motoyashiki, Kazuhiro Kohyama, Masataka Imura, Kunihiro Chihara: Fragra: A Visual-Olfactory VR Game.(2004) 